two were cases of recurrence after operation; nearly all were of long standing and complicated with chronic gastrointestinal disorders. For such cases electricity has not, in my hands at least, shown marked superiority over other and better known methods of treatment, although its use seems quite justifiable where operative treatment is positively refused.

6. Post-partum cases (really instances of partial rectal prolapse with feeble sphinater muscle).-Number of cases, 6. Number of applications, from 8 to 19. Result: Cured, 4 ; greatly relieved, 2. In all these cases the general tonic effect on the patient was quite as remarkable as the effect upon the local trouble.

7. Pruritus ani, with moist eczematous condition of the parts.-Number of cases, 15 . Number of applications, from 4 to 7 . Result: Cured in every case.

The above is a very brief outline of my results, but I trust that it may suffice to indicate in some measure the advantages and also the limitations of this variety of electrical treatment as applied to hæmorrhoids and other minor troubles of the rectum and anus. While the results as regards complete cures doubtless leave much to be desired they are still far superior to those obtained by any other local treatment short of operation. With experience also one is getting to select one's cases more carefully and there are many among those here recorded which I would not now undertake. It must also be remembered that hæmorrhoids are in many instances merely a symptom and result of hepatic or gastro-intestinal troubles. Where that is the case it will be idle to expect anything like a radical cure so long as the exciting cause remains.

Devonshire-street, $W$

\section{ACCIDENTAL VACCINIA OF THE NASAL CAVITY.}

By W. H. BOWEN, M.B., B.S. Lond., F.R.C.S. ENG., LATE CLINICAL ASSISTANT IN THE THROA'T DEPARTMENT, GUY'S HOSPITAL.

THE following case is thought worthy of publication on account of the rarity of the condition as shown by previously reported cases, of which I have only been able to find one (vide infra).

A married woman, aged 28 years, attended the throat department for out-patients at Guy's Hospital on April 22nd, 1904, for a "sore nose and inflamed cheek." Her history was as follows. Her last baby had been vaccinated 14 days previously and had "taken well." On the 19th, 11 days after the vaccination of the infant and four days before I first saw her, she felt her nose to be "sore and itching." The same evening the right ala of the nose and the adjacent part of the cheek and upper lip began to swell and "lumps" began to form beneath the lower jaw on the same side. There was no discharge from the nose. On the 20th the nose was very painful and a discharge like a lot of water came from the right nostril and the face became more swollen. She went to the front surgery of the hospital, where it was found that the cedema of the face had spread so that both cheeks were swollen, red, tender to the touch, and felt to be "throbbing" by the patient. There was no definite limiting margin to the swelling. 'The upper and lower lids of the right eye were so cdematous as to meet over the eyeball, but on separation the conjunctiva was found unaffected. The glands in the submaxillary region were greatly enlarged and tender. Although she "went off her food" she was not sick and could do her housework. Her temperature while in the front surgery is said to have been $98^{\circ} \mathrm{F}$. She was given hot boric fomentations to be used every four hours. On the 21st she felt much worse and could not eat or do her housework. The pain in the swollen face was worse and when she came to the hospital erysipelas was seriously discussed. She also complained on this day of something running down into the back of the throat which had a nasty smell.

In the absence of Mr. F. J. Steward I saw her on the 22nd and found the condition as follows. She was a strong, healthy looking woman. The right cheek was very much swollen, including the lower lid, the right side of the nose, and the whole of the upper lip, spreading back to the temporal region and tragus of the ear behind and below over the lower jaw, gradually fusing with a large submaxillary swelling due to the enlarged glands in this region. There was no appreciable enlargement of the glands in the anterior or posterior triangles of the neck. The mucous membrane of the mouth was perfectly normal. The swelling, she said, was much less than it had been and it was scarcely appreciable on the left cheek. This swelling was due to inflammatory œdema; it did not pit on pressure but was tender to the touch. The primary cause of the trouble was quickly found. Just within the right nostril on the floor and nearer the septum than the inferior meatus was a linear ulcer, perhaps a quarter of an inch in length, with its long axis from before back. This appearance was due to a swelling of unbroken mucous membrane on either side of a linear excoriation. It showed no characteristic signs at all and even when the mucus, of which there was a great quantity, had been cleared away it would have been extremely difficult to decide upon its nature from its appearance. Fortunately additional evidence was present in the form of a small greyish area situated just below the columna of about the size of a large pin-head surrounded by a red areola. This was, undoubtedly, the result of an accidental vaccination, and I had no doubt at all that the intranasal condition was of the same nature. As mentioned above her baby had been vaccinated a fortnight before. She denied, however, that the vesicles on the baby's arm had ever been broken and said that the scabs which had formed were still intact. She never remembered the baby scratching her with its nails, but she was suckling the infant. Anyone who has watched a baby either taking the breast or being carried in the mother's lap, remembering the restless way in which babies "claw" at everything about and with special frequency the mother's face, will readily understand the ease with which inoculation is brought about. The mother had not been vaccinated since she was a baby. She was advised to continue the hot boric fomentations to the right side of the face and was ordered chinosol, 1 in 1000 , to syringe the right naris with occasionally. Mr. Steward saw her on the next day and agreed with the diagnosis. On this day the intranasal infection had the greyish surface which, as pointed out by $\mathrm{Mr}$. Steward at the time, is almost typical of the condition. I watched the patient subsequently until the $26 \mathrm{th}$, when the vesicle inside the nose was scabbing over, and realising that she was better she did not attend further.

The only other case which I could find of accidental vaccinia of the nasal cavity was reported in the Birmingham Medical Review for 1903 (vol, i., p. 390) under the title of "Two Cases of Accidental Vaccination," by Dr. E. A. Sadler. In this case there were fissures found at the right ala of the nose. The patient was a woman, aged 32 years, and her baby had been vaccinated three weeks before. The mother had not bèen vaccinated since infancy.

Of 46 other cases of accidental vaccination which $I$ found reported in various journals, 31 were on the face. (Of these there were on the cheek, seven; on the eyelids or conjunctiva, or both, 11; on the chin, two; and on the lips, three.) One case is reported on the tongue, eight were situated on the trunk (including the breast, the buttocks, the penis, and the vulva), and four on the limbs. The diagnosis has usually been easy owing to the typical appearance of the vesicles. As might be expected it occurs far more frequently in women than in men, but often occurs in children, especially those who sleep with recently vaccinated brothers or sisters but have themselves either never been vaccinated or hare never "taken," whilst it is not infrequent in young girls who nurse a recently vaccinated baby and not having been revaccinated themselves are liable to feel the full effects of reinoculation. The signs and symptoms are similar to those following the ordinary inoculation of the arm, but one case is reported of the loss of the eye from accidental inoculation of the conjunctiva. The inoculation is frequently multiple though seldom more than three vesicles form. One extraordinary case is reported of inoculation on eczematous surfaces where 400 separate vesicles were found. This occurred in a boy, aged two years, whose infant brother had recently been vaccinated. The two children played and slept together. The constitutional symptoms in this case were not very marked.

Below is appended a list of references to cases of accidental vaccination. No case of accidental vaccinia of the nose was found either in the Proceedings of the Laryngological Society or in the Journal of Laryngology, Rhinology, and Otology.

Bibliography.-Abraham: Transactions of the Clinical Society 1896, 29, p. 240 (eyelid and chin). Bill: Illustrated Medical 

News, Nov, 17 th, 1888 (chin). Berry : Brit. Med. Jour., 1890,
vol. i., p. 1483 (five cases of vaccinia of eyelids). Buckell : Brit. vol. i., p. 1483 (five cases of vaceinia of eyelids). Buckell : Brit.
Med. 'Jour., 1889, vol. i., p. 1405 (dorsum of tongue). Buist: Transactions of the Edinburgh Obstetrical Society, 1890-91, vol. xvi., pp. 107-109 (three cases, including inoculation of exterior of nose, the chin, and the cheek). Doidge : Illustrated Medical News, Nov. 17th, 1888 (chin). Eminson: Brit. Med. Jour., 1888, vol. ii.,p. 1216 (vulva). Felkin : Transactions of the Edinburgh Obstetrical Society, 1890-91, vol. xvi., pp. 107-109 (nine cases reported, including labium, eye (2), cheek, breast, buttocks, lip, angle of mouth, and cheek (2) ). Fischer: Pediatrics (New York). 1897, vol. iii., p. 575 (cheek). Gottheil Archives of Pediatrics (New York), 1897, vol. xiv., pp. 849-51 (thigh) Kilham: Quarterly Medical Journal, Sheffield, vol, iii., p. 264 (cheek). Lamb: THE LANCET, Jan. 1st, 1898, p. 29 (penis and vulva in husband and wife). Makeig Jones: Quarterly Medical Journal, Sheffield, 1896-97, vol. v., p. 148 (arm, inoculated from cow). Mitehell: Australasian Medical Gazette, 1886-87, vol. vi., p. 140 (400 separate vesicles on eczematous surfaces; case referred to in text). Page: Brit. Med. Jour., 1888, vol. ii., p. 935 (cheek). Reid: Illustrated Medical News, Nov. 17th, 1888 (lower lip). Rutherford: Quarterly Medical Journal, Sheffield, 1896, vol. iv., p. 375 (eyelids and conjunctiva). Sadler: Birmingham Medical Review, 1903, New Series, vol. I, p. 390 (nose (1) and lower lip (2) ). Steel : THE LANCET, Jan. 24th, 1891, p. 191 (three Jour., 1892, vol. i., p. 115 (finger).

\section{THE PRESENCE OF BACTERIA IN THE ORGANS OF HEALTHY ANIMALS.}

BX H. DE R. MORGAN, M.A. Oxon., M.R.C.S. ENG., L.R.C.P. LOND., D.P.H. CANTAB.

From the Bacteriological Department of the Lister Institute of Preventive Medioine.)

THE possible occurrence of bacteria in the organs of normal animals has been made on many occasions a subject of inquiry. The question is obviously an important one whether considered clinically or scientifically. The interior of the healthy organism has usually been regarded as free from bacteria and the researches of Pasteur and of Duclaux have supported this conclusion. A number of observers have brought forward additional and confirmatory evidence. On the other hand, a recent research published by Ford ${ }^{1}$ furnishes, according to this author, experimental proof that normal organs do, as a matter of fact, contain bacteria. The paper gives a valuable résumé of the literature on the subject and from this I will quote before dealing with the author's own experiments. Meissner found no bacteria capable of development in the tissues of the healthy animal, whilst $Z$ weifel concluded that germs were habitually present but of an anaerobic character and limited in their capacity for development by the amount of oxygen in the blood stream. The researches of Hauser led to a negative result, the microscopic examination and the cultures made from healthy tissues showing them to be free from bacteria in every case. Welch only found the colon bacillus in the internal organs in cases of distinct lesion of the intestinal mucosa and he further states that this bacillus does not invade the blood and organs during post-mortem decomposition. Neisser, employing the usual precautions to avoid contamination, removed the liver, spleen, kidneys, heart, lungs, and mesentery from rabbits and guinea-pigs and placed the tissues in nutritive media. The subcultures made from these at the end of two days were invariably found to be sterile. Neisser concludes "that under normal conditions no bacteria are present in the lymph or blood stream." He found, on the other hand, that after producing artificial lesions of the intestines and feeding the animals experimented on with cultures of various micro-organisms the organs of such animals contained either these bacteria or representatives of the intestinal flora. Opitz carried out experiments on cattle in the abattoir at Breslau. Portions of the mesenteric glands from cattle were carefully sterilised on the surface and small pieces were then excised and cultivated upon agar and gelatin plates. It was found that at the end of three days no bacteria had developed in the majority of the tissues tested whilst those which were isolated from a small number of decomposed tissues were spore-bearing organisms-e.g., bacillus subtilis. Opitz concluded that the mesenteric glands of cattle are normally sterile and that bacteria do not pass through the healthy intestinal wall during digestion. Ford emphasises the fact that in his personal experiments the incubation of the tissues removed by him was prolonged

1 The Bacteriology of Normal Organs, Journal of Hygiene, 1901. for 17 days and that organisms were found to grow in the cultures as late as the seventeenth day. Neisser and Opitz, on the other hand, drew their conclusions from specimens which had only been incubated for a period of three days. Ford in a series of experiments made with 30 organs removed from rabbits found that 66 per cent. contained bacteria cultivable on agar, gelatin, and broth, whilst with 18 organs removed from guinea-pigs positive results were obtained in 61 per cent. The bacteria found in each case were identical and appeared in the culture media on the seventh day. It was thus only after a lapse of seven days that the organisms detected by Ford escaped from the organs which contained them and appeared as a growth in the culture soil. The bacteria found were staphylococcus albus and aureus, bacillus mesentericus, bacillus proteus, bacillus subtilis, and bacillus coli communis.

The organs of cats showed a bacterial flora different from that of the rabbits and guinea-pigs. Of 18 organs from the cat positive results were obtained in about 77 per cent. Staphylococci and colon bacilli were detected but not the bacillus subtilis or the bacillus mesentericus. The bacillus proteus, bacillus megatherium, and bacillus mycoides were found as well as two spore-bearing bacilli detected in the broth cultures. These spore-bearing forms along with the bacillus megatherium and bacillus mycoides and at times bacillus Zopfii were also present in the organs of dogs. The experiments of Ford showed that out of 96 organs taken from 34 animals 69 per cent. contained bacteria and that less than 30 per cent. were sterile. It appeared advisable in view of the exceptional character of these results to repeat the experiments and I will now briefly describe the methods which I adopted and the results. Every precaution was taken in the course of the experiments to avoid aerial or other accidental contamination. A rabbit was killed, the skin was deflected from a central linear incision, and the abdominal muscles were seared with a red-hot iron. The abdomen was opened with a sterilised scalpel and ten cubic centimetres of peptone broth were poured over the intestines and immediately drawn up, together with any peritoneal fluid, by means of a sterile syringe. The fluid obtained in this way was placed in a sterile tube for subsequent cultivation. The intestines were next drawn aside by sterile glass rods and the exposed organs or portions were removed-viz., the kidney, the suprarenals, the spleen, the liver, and a few of the mesenteric glands. Each organ was quickly passed through the flame of a Bunsen burner and then divided, one portion being dropped into a flask of nutrient broth and the other into melted agar cooled down to $45^{\circ} \mathrm{C}$. The organs in the broth were broken up with a sterilised knife and both broth and agar were incubated at blood heat. The broth cultures were employed to test for aerobic and the agar cultures for the growth of anaerobic micro-organisms. After 17 days' incubation portions of the organs planted in the agar were removed with a platinum needle, transferred to broth tubes, and kept for several days at blood heat under anaerobic conditions in a modified Buchner's tube and eventually examined for bacterial growth. From the organs in broth, subcultures were made in fresh broth every third day, care being taken to avoid contamination. These subcultures were kept at blood heat, and in the event of no growth occurring were reinoculated on the second or third day. 80 organs and the peritoneal fluid from ten rabbits were tested in the above manner. Growths were obtained from 23 organs in the aerobic broth cultures and from one rabbit two organs gave a growth of the colon bacillus. This latter result was, however, probably due to an accidental injury to the intestine and a consequent contamination of the surface of the organs. In no case was a growth of the colon bacillus obtained from an animal with uninjured intestines. The bacteria present in the broth cultures were non-pathogenic forms and their intraperitoneal injection in rabbits proved harmless. They consisted mostly of such common types as the bacillus mesentericus vulgatus along with a number of other non-pathogenic spore-bearing forms. These organisms never appeared later than the third day in the cultures and differed in no respect from similar organisms planted per se in peptone broth. From the anaerobic agar cultures no growth was obtained in any instance and I was unable to confirm Zweifel's statement as to the presence of anaerobio organisms in the healthy body tissues. Similar experiments were made with seven organs from the cat and a similar number from the dog. Aerobic growths of non-pathogenic forms were obtained in three out of seven organs in each case. 